\title{
Spatially adaptive alpha-rooting in BM3D sharpening
}

\author{
Markku Mäkitalo and Alessandro Foi \\ Department of Signal Processing, Tampere University of Technology, \\ P.O. Box FIN-553, 33101, Tampere, Finland e-mail: firstname.lastname@tut.fi
}

\begin{abstract}
The block-matching and 3-D filtering (BM3D) algorithm is currently one of the most powerful and effective image denoising procedures. It exploits a specific nonlocal image modelling through grouping and collaborative filtering. Grouping finds mutually similar 2-D image blocks and stacks them together in 3-D arrays. Collaborative filtering produces individual estimates of all grouped blocks by filtering them jointly, through transform-domain shrinkage of the 3-D arrays (groups).

BM3D can be combined with transform-domain alpha-rooting in order to simultaneously sharpen and denoise the image. Specifically, the thresholded 3-D transform-domain coefficients are modified by taking the $\alpha$-root of their magnitude for some $\alpha>1$, thus amplifying the differences both within and between the grouped blocks. While one can use a constant (global) $\alpha$ throughout the entire image, further performance can be achieved by allowing different degrees of sharpening in different parts of the image, based on content-dependent information.

We propose to vary the value of $\alpha$ used for sharpening a group through weighted estimates of the lowfrequency, edge, and high-frequency content of the average block in the group. This is shown to be a viable approach for image sharpening, and in particular it can provide an improvement (both visually and in terms of PSNR) over its global non-adaptive counterpart.
\end{abstract}

\section{INTRODUCTION}

In image processing, sharpening refers to enhancing the details, such as edges and textures, of an image for any particular purpose. The aim can be, for instance, to produce a visually more pleasing image, but it can also serve as a preliminary step for further processing or analysis, such as pattern recognition or classification, when the features of interest are blurry or portrayed with insufficient contrast. In practice, images are corrupted by noise, so typically it is desirable for the sharpening algorithm to also suppress the noise while sharpening the image details.

The block-matching and 3-D filtering (BM3D) algorithm ${ }^{1}$ is currently one of the most powerful and effective image denoising procedures. ${ }^{2,3}$ It exploits a specific nonlocal image modelling through the procedures of grouping and collaborative filtering. Grouping finds mutually similar 2-D image blocks and stacks them together in 3-D arrays. Collaborative filtering produces individual estimates of all grouped blocks by filtering them jointly, through transform-domain shrinkage of the 3-D arrays (groups). In doing so, BM3D relies both on nonlocal and local characteristics of natural images, namely the abundance of mutually similar patches and the fact that image data is locally highly correlated. Because of this, the group enjoys correlation in all three dimensions and a sparse representation of the true signal is obtained by applying a decorrelating 3-D transform on the group. As BM3D typically produces several estimates for a particular block, their weighted linear combination forms the final estimate of a block in the final aggregation step of the algorithm.

In Ref. 4, BM3D is combined with transform-domain alpha-rooting ${ }^{5}$ in order to simultaneously sharpen and denoise the image. Specifically, the thresholded 3-D transform-domain coefficients $t$ are modified by taking the $\alpha$-root of their magnitude for some $\alpha>1$, thus amplifying the differences both within and between the grouped blocks:

$$
t_{\alpha}(i)= \begin{cases}\operatorname{sign}[t(i)]|t(0)|\left|\frac{t(i)}{t(0)}\right|^{\frac{1}{\alpha}}, & \text { if } t(0) \neq 0 \\ t(i), & \text { otherwise, }\end{cases}
$$

where $t_{\alpha}$ is the resulting alpha-rooted 3-D spectrum, and $t(0)$ is the DC component of the spectrum.

While, as was done in Ref. 4, one can use a constant (i.e., global) $\alpha$ throughout the entire image, further performance can be achieved by allowing different degrees of sharpening in different parts of the image, based on content-dependent information. This is the basic motivation for spatially adaptive alpha-rooting. 


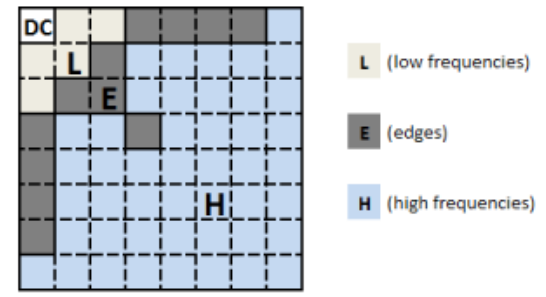

Figure 1. The division of the $8 \times 82$-D DCT coefficients into four categories: DC, low frequencies $(L)$, edges $(E)$, and high frequencies $(H) .^{6}$

\section{SPATIALLY ADAPTIVE ALPHA-ROOTING}

In this paper, we assume to have an image observation $z=\widetilde{y}+\eta$, where $\widetilde{y}$ is an image that is noise-free but poor in contrast and lacking sharpness, and $\eta$ is additive white Gaussian noise with zero mean and variance $\sigma^{2}$. We denote the unknown ideal sharp image by $y$.

In alpha-rooting, the value $\alpha=1$ corresponds to no sharpening. It is reasonable to assume that the smooth areas of the image do not require strong sharpening. However, for edges and textures the amount of sharpening to be performed should be relatively higher. We propose to vary the value of $\alpha$ used for sharpening a group through weighted estimates of the edge and texture content of the average block in the group. For this purpose, we follow the approach by Tong and Venetsanopoulos, ${ }^{6}$ who have proposed to estimate the content of $8 \times 8$ image blocks from their DCT coefficients by dividing them into four categories: DC, low-frequency, edge, and high-frequency coefficients. The division is illustrated in Figure 1 . The $\ell_{1}$-norm of the coefficients in each category produces values $D C, L, E$ and $H$, respectively. The texture strength can be approximated by the sum $E+H$.

Provided that the $3-\mathrm{D}$ transform used by the BM3D algorithm is realized as a separable composition of the 2-D DCT and 1-D Haar transforms, one 2-D plane of the 3-D spectrum is (up to a scaling factor) exactly the 2-D DCT spectrum of the average block in the group. This makes the approach of Tong and Venetsanopoulos practical. In particular, we implement it in the following way. After noise has been suppressed by hard-thresholding of the 3-D spectrum, we compute the $E, L$ and $H$ values from the thresholded coefficients of the 2-D plane, as described above. Then, for the alpha-rooting, we propose an affine model for adaptively varying the parameter $\alpha$ as a function of $E, L$ and $H$ :

$$
\alpha_{\left(\omega_{1}, \omega_{2}, \omega_{3}\right)}(E, L, H)=1+\omega_{1} \cdot E+\omega_{2} \cdot L+\omega_{3} \cdot H
$$

where $\omega_{1}, \omega_{2}, \omega_{3} \in \mathbb{R}$ are weights for the relative impact of edge, low-frequency, and high-frequency contents in the average block, respectively. In our experiments we evaluate the performance of this sharpening technique, examine the choice of the weights $\omega_{i}$ with the aid of an oracle, and then discuss how the computed weights can be applied in practical cases.

It is worth noting that for simplicity we restrict our scope to an affine model, and acknowledge the fact that introducing more complex nonlinear functions into the parametric model is a likely source for further improvements.

\section{EXPERIMENTS}

In Section 3.1, we first train our sharpening algorithm by fitting the weights of the model (2) for a particular image blurred with a specific Gaussian point-spread function (PSF). Then we investigate the sharpening performance of the spatially adaptive alpha-rooting with the fitted parameters, for the same image. In Section 3.2, we then examine the general applicability of these fitted weights for sharpening other images corrupted by different PSFs (i.e., for new information that was not available during the training). For all the experiments, apart from using the $8 \times 82$-D DCT within the 3-D transform, all other elements and parameters of the algorithm are as in the standard implementation with fixed $\alpha .^{4}$ 


\subsection{Fitting the weights $\omega_{i}, i=1,2,3$}

In this section, we use a compound image $y$ of size $768 \times 512$, obtained by tiling the Cameraman, Peppers and Lena images (Figure 2). We blur this image by convolving it $n$ times $(n=1,2,3,4)$ with the PSF

$$
K_{1}=\frac{1}{16}\left[\begin{array}{lll}
1 & 2 & 1 \\
2 & 4 & 2 \\
1 & 2 & 1
\end{array}\right]
$$

resulting in $\widetilde{y}$, and then add white Gaussian noise $(\sigma=5,10,15,20,25,30,35,40,45,50)$, for a total of 40 different combinations of noise and blur. We denote convolution by the $\otimes$ symbol; for instance, blurring an image with $K_{1} \otimes K_{1}$ means the image is convolved twice (i.e., $n=2$ ) with the PSF $K_{1}$. Note that throughout the experiments, we remove 10 pixels from each edge of every image before doing any further processing, such as fitting the weights, sharpening and computing the PSNR values, in order to prevent border effects caused by the convolution from affecting the results.

First, we directly compute the optimal group-wise values for the sharpening parameter $\alpha$ (without using any parametric model, such as (2)). In order to compensate for the effect of noise on the sharpening parameter, we provide this oracle not only with the original image, but also with the blurred, but noise-free, image $\widetilde{y}$. Then, for each group inside the spatially adaptive alpha-rooting BM3D algorithm, we search for the $\alpha$ that minimizes the mean square error (MSE) between the alpha-rooted 3-D transform coefficients of the blurry noise-free group and the corresponding spectrum of the original group, instead of comparing the spectrum of the group that is both noisy and blurry against the original spectrum. Note that this choice is due to the fact that in practice the aggregation step (weighted averaging) in BM3D provides us with effective noise suppression. More precisely, for each group we choose

$$
\alpha^{*}=\underset{\alpha}{\arg \min }\left\|t_{\alpha}^{(\widetilde{y})}-t^{(y)}\right\|_{2}^{2},
$$

where $t_{\alpha}^{(\widetilde{y})}$ is the alpha-rooted (unthresholded) 3-D spectrum of the blurred noise-free group, and $t^{(y)}$ the corresponding 3-D spectrum of the original group.

This oracle result ("spatially varying oracle") gives us some indication of the potential of spatially adaptive alpha-rooting. However, it should be noted that this oracle does not necessarily correspond to the highest attainable PSNR, as it operates group-wise instead of minimizing the global MSE. Moreover, it is good to keep in mind that the oracle search does not guarantee optimal visual results, as MSE and PSNR do not correlate perfectly with the subjective visual quality.

Then, in order to investigate the performance of our parametric model (2), we fit the weights $\omega_{i}, i=1,2,3$, of the affine parametric model (2) for the sharpening parameter $\alpha$, separately for each of the 40 combinations of noise and blur. This is done by optimizing the weights in such a way that the total sum of all group-wise MSE values is minimized:

$$
\left(\omega_{1}, \omega_{2}, \omega_{3}\right)=\underset{\omega_{1}, \omega_{2}, \omega_{3}}{\arg \min } \sum_{G} \frac{1}{j_{G}}\left\|t_{\alpha_{\left(\omega_{1}, \omega_{2}, \omega_{3}\right)}(E, L, H)}^{(\widetilde{y})}-t^{(y)}\right\|_{2}^{2},
$$

where the sum is taken over all groups $G$ processed by the BM3D algorithm, and $j_{G}$ is the number of elements in the 3-D spectrum of group $G$. As earlier, each MSE value is computed between the alpha-rooted 3-D transform coefficients $t_{\alpha}^{(\widetilde{y})}$ of the blurry noise-free group and the corresponding spectrum $t^{(y)}$ of the original group. However, now our choice of $\alpha$ is not entirely free: for each group of the noisy observation $z$, the $E$, $L$ and $H$ values are computed from the average DCT coefficients, and the corresponding $\alpha_{\left(\omega_{1}, \omega_{2}, \omega_{3}\right)}(E, L, H)$ is then obtained according to $(2)$.

For further comparison, we also search for the optimal fixed value of $\alpha$ that produces the highest PSNR, when the image is sharpened with the non-adaptive BM3D sharpening algorithm. ${ }^{4}$ This optimal global $\alpha$ ("spatially invariant oracle") is typically rather small and decreases as the noise level $\sigma$ increases, because it is a compromise between sharpening the edges and not sharpening the smooth areas, as the latter would lead to unnecessary noise amplification.

The numerical results of sharpening the compound image with these different methods are presented in Table 1, and the fitted weights of the parametric model (2) corresponding to the fifth column of Table 1 are 


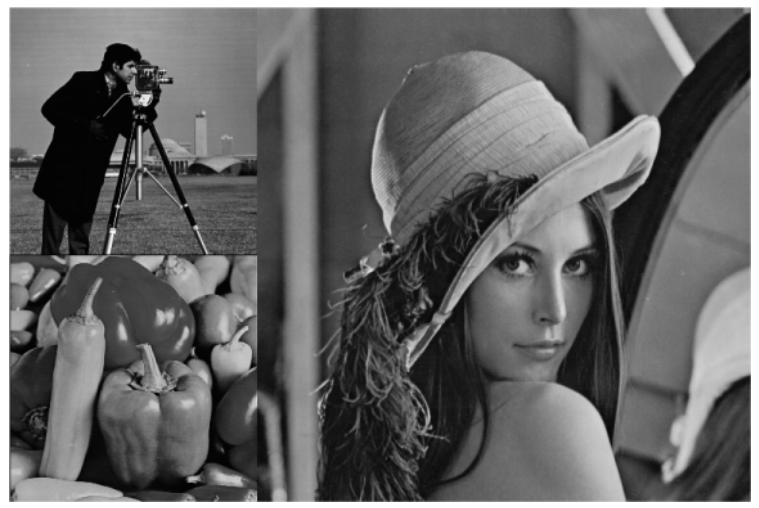

Figure 2. The original noise-free image $(768 \times 512)$, composed of the Cameraman, Peppers and Lena images.

further shown in Table 2. Besides choosing the weights $\omega_{i}$ of the model (2) based on the true value of $n=1,2,3,4$, we also examine the case $n=8$ by extrapolating the corresponding weights from the computed values. We notice that by overestimating the parameter $n$ we are in many cases able to exceed the PSNR result given by the spatially varying oracle; this is due to the difference in the minimization criteria (4) and (5) with respect to the global PSNR.

The values of the sharpening parameter $\alpha$ for the case $\sigma=10, n=3$ are visualized in Figures 3(a) and 3(b), which correspond to the spatially varying oracle and parametric cases, respectively. These spatial maps show that the parametric model is able to provide reasonable estimates for $\alpha$, even though the spatial map provided by the oracle is sharper and richer in detail.

\subsection{General applicability of the fitted weights}

In this section, we apply the fitted weights presented in Table 2 for sharpening other images (namely House and Boat, shown in Figures 4(a) and 4(b)) corrupted as in Section 3.1, and with different PSFs:

$$
K_{2}=\left[\begin{array}{lll}
0.0113 & 0.0838 & 0.0113 \\
0.0838 & 0.6193 & 0.0838 \\
0.0113 & 0.0838 & 0.0113
\end{array}\right], \quad K_{3}=\left[\begin{array}{lllll}
0.0039 & 0.0078 & 0.0391 & 0.0078 & 0.0039 \\
0.0078 & 0.0156 & 0.0781 & 0.0156 & 0.0078 \\
0.0391 & 0.0781 & 0.3906 & 0.0781 & 0.0391 \\
0.0078 & 0.0156 & 0.0781 & 0.0156 & 0.0078 \\
0.0039 & 0.0078 & 0.0391 & 0.0078 & 0.0039
\end{array}\right] .
$$

Note that the latter kernel is obtained by setting $K_{3}=\frac{L^{T} L}{\left\|L^{T} L\right\|_{1}}$, where $L=\left[\begin{array}{lllll}0.1 & 0.2 & 1 & 0.2 & 0.1\end{array}\right]$.

For each case we use various values of the parameter $n$, resulting in different levels of sharpening. We remark that here $n$ relates neither to the PSF nor to the amount of blur present in $\widetilde{y}$. In particular, the structure and strength of the blur are not known. Estimating the noise variance is a relatively straightforward process, so we assume that this quantity is known to us. Thus, we perform sharpening with seven different values of $n$ ( $n=1,2,3,4,5,8,10)$ and using the corresponding weights in Table 2 ; the weights for the cases $n=5,8,10$ are linearly extrapolated from the fitted values for $n=3,4$.

Tables 3-8 present the sharpening results of the proposed spatially adaptive alpha-rooting method. For comparison, we also show the best result ("spatially invariant oracle", in terms of PSNR) obtained by the nonadaptive BM3D sharpening algorithm. ${ }^{4}$ As the amount of blur depends not only on the parameter $n$, but also on the structure of the PSF itself, the true $n$ is not relevant for choosing the weights from Table 2, especially if one wants to maximize the PSNR of the sharpened image; instead, the obtained results indicate that a large $n$ is generally beneficial. From another point of view, tuning the parameter $n$ provides a practical way to control the amount of sharpening to be performed on the image. Most importantly, the results show that even though the weights were fitted for a specific image and PSF, the proposed method can be applied to other images and other types of PSFs as well, and it can provide a clear improvement compared to non-adaptive alpha-rooting. In particular, its advantage in terms of PSNR tends to increase with the noise level $\sigma$. 


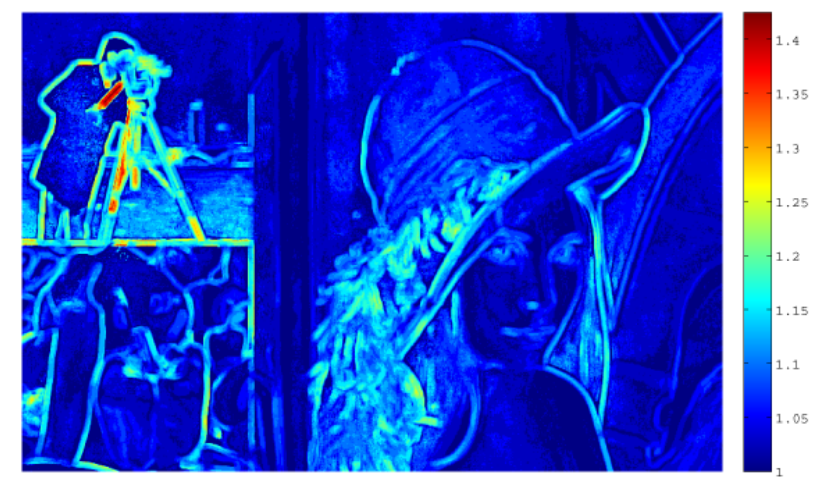

(a)

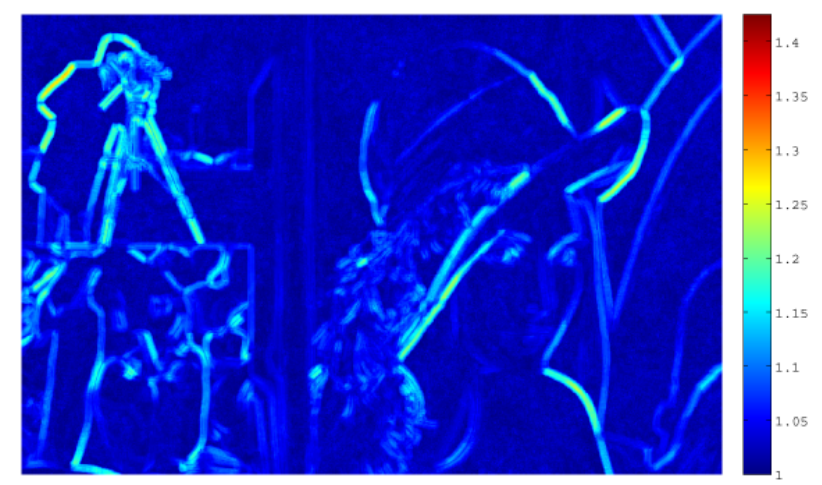

(b)

Figure 3. Spatial maps of the sharpening parameter $\alpha$ for the compound image, case $\sigma=10, n=3$. (a) Spatially varying oracle (nonparametric) values of $\alpha$, where $\alpha \in[1,1.4250]$. (b) Parametric model $\alpha=1+\omega_{1} \cdot E+\omega_{2} \cdot L+\omega_{3} \cdot H$, where the fitted weights are $\omega_{1}=0.1061, \omega_{2}=0.0352$ and $\omega_{3}=0.1542$, resulting in the range $\alpha \in[1,1.3022]$.

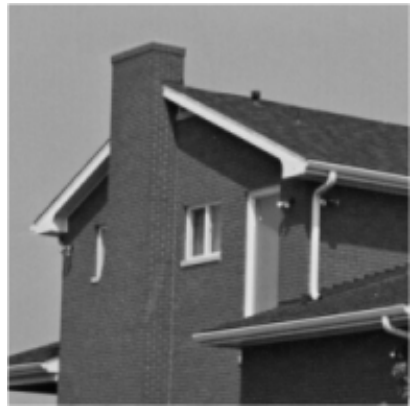

(a)

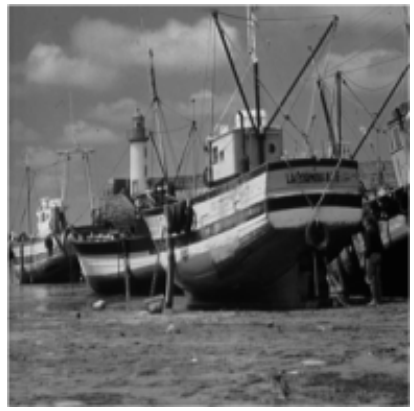

(b)

Figure 4. The original noise-free images used for the validation of the fitted model obtained in Section 3.1. (a) House $(256 \times 256)$. (b) Boat $(512 \times 512)$.

Visual comparisons of the proposed method and its non-adaptive counterpart are presented in Figures 5-6, which also confirm that spatially adapting the alpha-rooting strength is a viable method for image sharpening, and we see that it can provide us with more contrast and details than the non-adaptive method. Moreover, it is able to suppress the noise in smooth areas much more effectively. We also observe that the parameter value $n=10$ results in a sharper image than the one obtained with $n=5$, despite its slightly lower PSNR. Figure 7 illustrates the full spatial map of $\alpha_{\left(\omega_{1}, \omega_{2}, \omega_{3}\right)}(E, L, H)$ corresponding to the sharpening result of Figure 6(d) (i.e., $\sigma=30, n=8$; see also Table 7 ). 


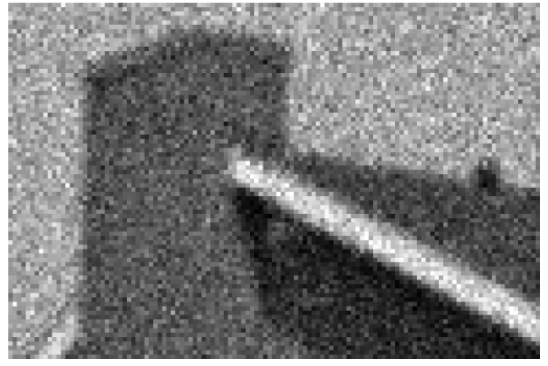

(a)

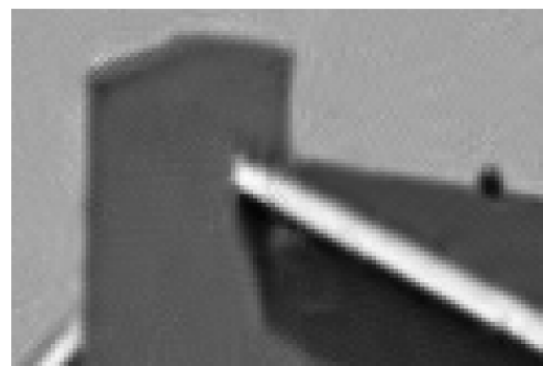

(c)

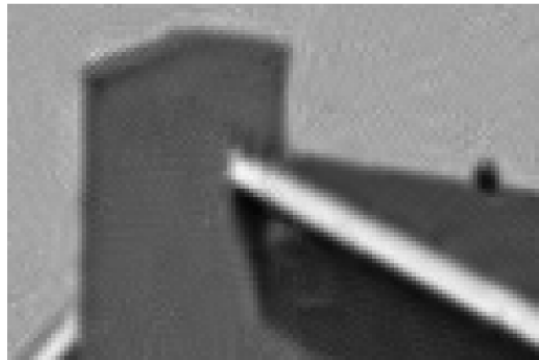

(b)

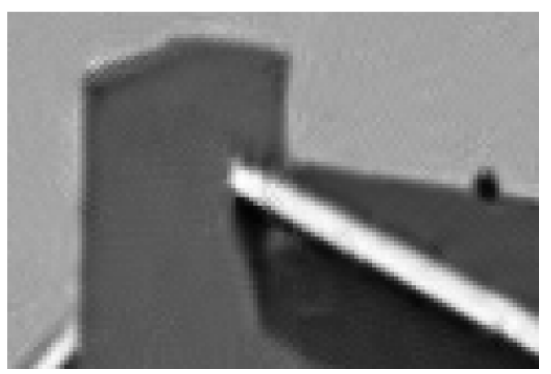

(d)

Figure 5. Comparison of the sharpening results. (a) An enlarged section of the noisy and blurry House $(\sigma=20$, blurred with the PSF $K_{1} \otimes K_{1}$ ). (b) Sharpened with global alpha-rooting ${ }^{4}$ (PSNR-optimal fixed $\alpha=1.125$, PSNR $=29.95 \mathrm{~dB}$ ). (c) Sharpened with the parametric model (2) using the linearly extrapolated weights for $n=5\left(\omega_{1}=0.1074, \omega_{2}=0.0679\right.$, $\omega_{3}=0.0990$, PSNR $\left.=30.13 \mathrm{~dB}\right)$. (d) Sharpened with the parametric model (2) using the linearly extrapolated weights for $n=10\left(\omega_{1}=0.1094, \omega_{2}=0.1395, \omega_{3}=0.1076\right.$, PSNR $\left.=30.01 \mathrm{~dB}\right)$. The reported PSNR values are for the full House image (see Table 3), but only one section of it is shown for better visualization of the differences.

As a final example, we sharpen an image featuring a longsnout seahorse, taken with a modern digital camera (Canon EOS 500D) but lacking in sharpness. For simplicity, we process only one of the three colour channels, namely the blue one (Figure 8(a)). In order to treat the noise as additive white Gaussian, we leverage the ClipPoisGaus package ${ }^{7}$ for data corrupted by signal-dependent noise, which automatically estimates the noise model parameters and stabilizes the variance through homomorphic transformations. The estimated standard deviation of the noise in the variance-stabilized image is $\sigma=2.1194$. Then we linearly extrapolate the weights $\omega_{i}$ for this particular $\sigma$ and $n=10$, and use the resulting parametric model $\alpha_{\left(\omega_{1}, \omega_{2}, \omega_{3}\right)}(E, L, H)$ for sharpening the variance-stabilized image. After the inverse homomorphic transformation, we obtain the image shown in Figure 8(b). The spatial map of $\alpha_{\left(\omega_{1}, \omega_{2}, \omega_{3}\right)}(E, L, H)$ is visualized in Figure $8(\mathrm{c})$. The clear improvement in sharpness and details, together with the absence of visible noise in the result, supports our earlier observations about the viability and potential of spatially adaptive alpha-rooting in image sharpening.

\section{DISCUSSION AND CONCLUSIONS}

We proposed a method for spatially adaptive selection of the alpha-rooting strength for each group inside the BM3D denoising algorithm, through weighted estimates of the low-frequency, edge, and high-frequency contents of the average block in the group. Our experiments showed that BM3D with spatially adaptive alpha-rooting is a viable approach for image sharpening, and in particular it can provide an improvement (both visually and in terms of PSNR) over its global non-adaptive counterpart. Since the $E, L$ and $H$ values are computed from the same spectrum given by the collaborative filtering, it introduces no significant computational overhead as compared to the procedure with a fixed global $\alpha$.

We fitted the weights $\omega_{i}$ for our affine model of the alpha-rooting parameter, and observed that the computed weights (either directly or through inter- and extrapolation) can be successfully applied to sharpening other images corrupted with a different PSF. We also observed that the amount of sharpening can be controlled by using different values for the parameter $n$, based on which the weights are chosen. 


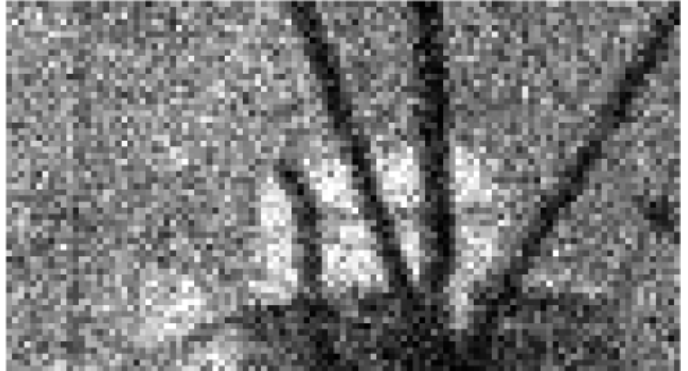

(a)

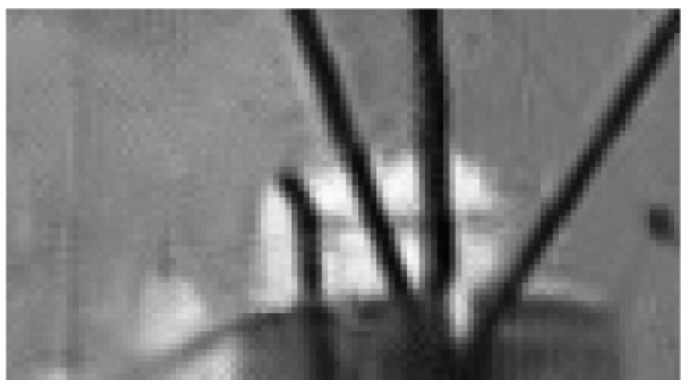

(c)

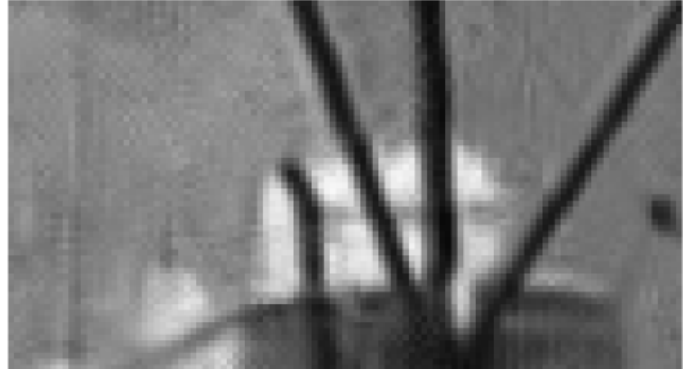

(b)

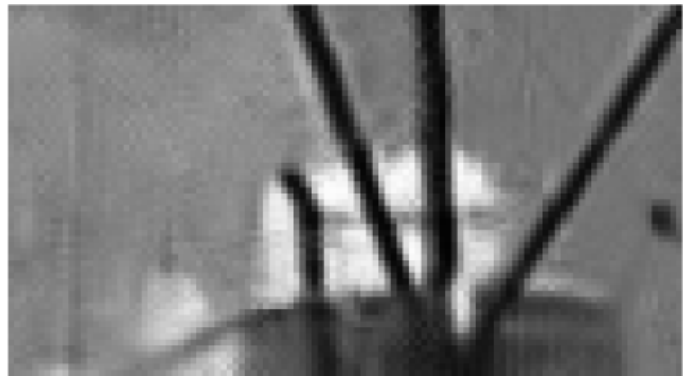

(d)

Figure 6. Comparison of the sharpening results. (a) An enlarged section of the noisy and blurry Boat ( $\sigma=30$, blurred with the PSF $K_{2} \otimes K_{2} \otimes K_{2}$ ). (b) Sharpened with global alpha-rooting ${ }^{4}$ (PSNR-optimal fixed $\alpha=1.100$, PSNR $=26.87 \mathrm{~dB}$ ). (c) Sharpened with the parametric model (2) using the weights for $n=4\left(\omega_{1}=0.0801, \omega_{2}=0.0557, \omega_{3}=0.0636\right.$, PSNR $=26.93 \mathrm{~dB})$. (d) Sharpened with the parametric model (2) using the linearly extrapolated weights for $n=8\left(\omega_{1}=0.0769\right.$, $\omega_{2}=0.1039, \omega_{3}=0.0688$, PSNR $=26.95 \mathrm{~dB}$ ). The reported PSNR values are for the full Boat image (see Table 7), but only one section of it is shown for better visualization of the differences.

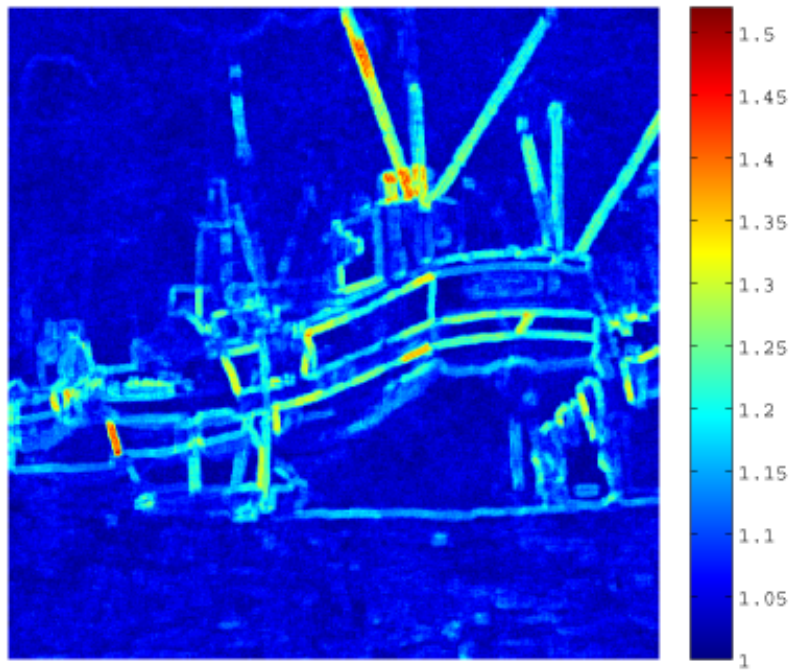

Figure 7. The full spatial map of the sharpening parameter $\alpha_{\left(\omega_{1}, \omega_{2}, \omega_{3}\right)}(E, L, H) \in[1,1.5205]$ corresponding to the result of Figure $6(\mathrm{~d})$ (i.e., $\sigma=30, n=8$; see also Table 7 ). 


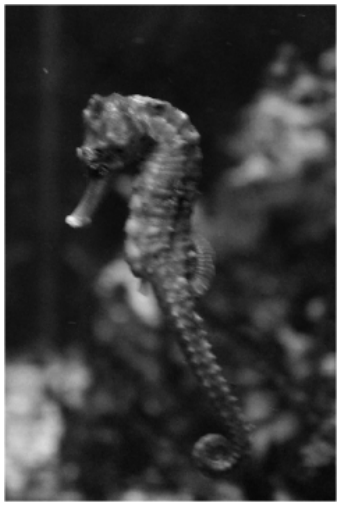

(a)

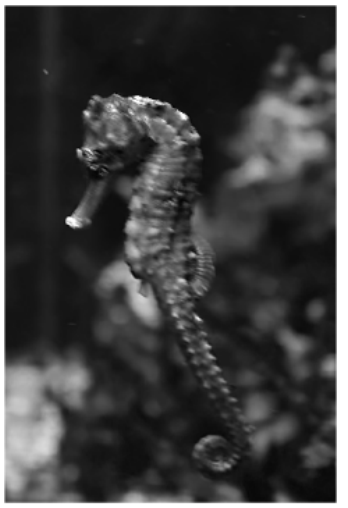

(b)

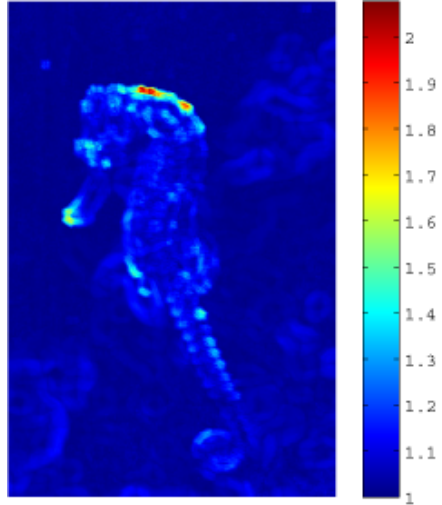

(c)

Figure 8. Sharpening of the Seahorse image. (a) The original image $(317 \times 476)$, lacking in sharpness. (b) Sharpened with the parametric model (2) using the linearly extrapolated weights for $n=10$ and the estimated $\sigma=2.1194\left(\omega_{1}=-0.0183\right.$, $\left.\omega_{2}=0.1408, \omega_{3}=0.6816\right)$. (c) The full spatial map of the sharpening parameter $\alpha_{\left(\omega_{1}, \omega_{2}, \omega_{3}\right)}(E, L, H) \in[0.9994,2.0774]$.

Overall, we have shown that the concept of spatially adaptive alpha-rooting has potential in image sharpening. We expect that applying both more sophisticated nonlinear parametric models and other edge/texture content indicators may lead to further improvement. As an example, the authors of Ref. 6 suggest that high values of the ratios $(L+E) / H$ and $L / E$ correspond well to edges.

\section{ACKNOWLEDGMENTS}

This work was supported by the Academy of Finland (project no. 213462, Finnish Programme for Centres of Excellence in Research 2006-2011, project no. 118312, Finland Distinguished Professor Programme 2007-2010, and project no. 129118, Postdoctoral Researchers Project 2009-2011) and by Tampere Doctoral Programme in Information Science and Engineering (TISE).

\section{REFERENCES}

1. Dabov, K., A. Foi, V. Katkovnik, and K. Egiazarian, "Image denoising by sparse 3D transform-domain collaborative filtering", IEEE Trans. Image Process., vol. 16, no. 8, pp. 2080-2095, Aug. 2007.

2. Chatterjee, P., and P. Milanfar, "Is Denoising Dead?", IEEE Trans. Image Processing, vol. 19, no. 4, pp. 895-911, April 2010.

3. Katkovnik, V., A. Foi, K. Egiazarian, and J. Astola, "From local kernel to nonlocal multiple-model image denoising", Int. J. Computer Vision, vol. 86, no. 1, pp. 1-32, January 2010.

4. Dabov, K., A. Foi, V. Katkovnik, and K. Egiazarian, "Joint image sharpening and denoising by 3D transformdomain collaborative filtering", Proc. 2007 Int. TICSP Workshop Spectral Meth. Multirate Signal Process. (SMMSP 2007), Moscow, Russia, Sept. 2007. Software available online at http://www.cs.tut.fi $/{ }^{\sim}$ foi $/$ GCFBM3D.

5. Aghagolzadeh, S., and O. K. Ersoy, "Transform image enhancement", Opt. Eng., vol. 31, no. 3, pp. 614-626, March 1992.

6. Tong, H.H.Y., and A.N. Venetsanopoulos, "A perceptual model for JPEG applications based on block classification, texture masking, and luminance masking", 1998 International Conference on Image Processing (ICIP'98), vol. 3, 1998.

7. Foi, A., "Clipped noisy images: heteroskedastic modeling and practical denoising", Signal Processing, vol. 89, no. 12, pp. 2609-2629, Dec. 2009. doi:10.1016/j.sigpro.2009.04.035. "ClipPoisGaus" Matlab software available online at http://www.cs.tut.fi/ foi/sensornoise. 
Table 1. PSNR (dB) of the sharpened compound image, sharpened either with nonparametric spatially adaptive alpharooting ("spatially varying oracle"), optimal non-adaptive alpha-rooting ("spatially invariant oracle"), or spatially adaptive alpha-rooting with the parametric alpha (2). In the first parametric case, the fitted weights $\omega_{i}$ of the model are chosen based on the true value of $n=1,2,3,4$, and in the second parametric case they are extrapolated for $n=8$.

\begin{tabular}{|c|c|c|c|c|c|}
\hline$\sigma$ & $n$ (blur) & $\begin{array}{c}\text { Spatially varying } \\
\text { oracle }\end{array}$ & $\begin{array}{l}\text { Spatially invariant } \\
\text { oracle }\end{array}$ & $\begin{array}{l}\text { Fitted model }(2), \\
\text { true } n=1,2,3,4\end{array}$ & $\begin{array}{c}\text { Fitted model (2), } \\
n=8\end{array}$ \\
\hline \multirow{4}{*}{5} & 1 & 32.03 & $31.87(\alpha=1.100)$ & 31.73 & 30.25 \\
\hline & 2 & 30.19 & $30.09(\alpha=1.150)$ & 29.84 & 30.17 \\
\hline & 3 & 29.12 & $29.07(\alpha=1.200)$ & 28.75 & 29.25 \\
\hline & 4 & 28.36 & $28.37(\alpha=1.225)$ & 27.99 & 28.39 \\
\hline \multirow{4}{*}{10} & 1 & 30.86 & $30.74(\alpha=1.100)$ & 30.64 & 30.00 \\
\hline & 2 & 29.36 & $29.26(\alpha=1.150)$ & 29.10 & 29.43 \\
\hline & 3 & 28.46 & $28.37(\alpha=1.175)$ & 28.18 & 28.58 \\
\hline & 4 & 27.80 & $27.74(\alpha=1.225)$ & 27.51 & 27.84 \\
\hline \multirow{4}{*}{15} & 1 & 29.93 & $29.82(\alpha=1.100)$ & 29.76 & 29.64 \\
\hline & 2 & 28.66 & $28.55(\alpha=1.150)$ & 28.46 & 28.76 \\
\hline & 3 & 27.87 & $27.76(\alpha=1.175)$ & 27.65 & 27.96 \\
\hline & 4 & 27.29 & $27.19(\alpha=1.200)$ & 27.06 & 27.32 \\
\hline \multirow{4}{*}{20} & 1 & 29.12 & $29.01(\alpha=1.100)$ & 28.99 & 29.03 \\
\hline & 2 & 28.03 & $27.90(\alpha=1.150)$ & 27.87 & 28.13 \\
\hline & 3 & 27.34 & $27.19(\alpha=1.175)$ & 27.16 & 27.42 \\
\hline & 4 & 26.82 & $26.67(\alpha=1.175)$ & 26.64 & 26.84 \\
\hline \multirow{4}{*}{25} & 1 & 28.41 & $28.29(\alpha=1.100)$ & 28.31 & 28.42 \\
\hline & 2 & 27.47 & $27.31(\alpha=1.125)$ & 27.34 & 27.55 \\
\hline & 3 & 26.85 & $26.68(\alpha=1.150)$ & 26.70 & 26.90 \\
\hline & 4 & 26.39 & $26.20(\alpha=1.150)$ & 26.23 & 26.39 \\
\hline \multirow{4}{*}{30} & 1 & 27.79 & $27.66(\alpha=1.075)$ & 27.71 & 27.82 \\
\hline & 2 & 26.95 & $26.78(\alpha=1.100)$ & 26.84 & 27.01 \\
\hline & 3 & 26.40 & $26.20(\alpha=1.125)$ & 26.27 & 26.42 \\
\hline & 4 & 25.98 & $25.78(\alpha=1.125)$ & 25.85 & 25.97 \\
\hline \multirow{4}{*}{35} & 1 & 27.21 & $27.06(\alpha=1.075)$ & 27.14 & 27.23 \\
\hline & 2 & 26.47 & $26.29(\alpha=1.075)$ & 26.38 & 26.51 \\
\hline & 3 & 25.97 & $25.77(\alpha=1.100)$ & 25.87 & 25.98 \\
\hline & 4 & 25.60 & $25.38(\alpha=1.100)$ & 25.49 & 25.58 \\
\hline \multirow{4}{*}{40} & 1 & 26.56 & $26.43(\alpha=1.025)$ & 26.50 & 26.52 \\
\hline & 2 & 25.95 & $25.77(\alpha=1.050)$ & 25.86 & 25.93 \\
\hline & 3 & 25.53 & $25.33(\alpha=1.050)$ & 25.43 & 25.50 \\
\hline & 4 & 25.20 & $24.99(\alpha=1.075)$ & 25.10 & 25.16 \\
\hline \multirow{4}{*}{45} & 1 & 26.25 & $26.19(\alpha=1.075)$ & 26.19 & 26.33 \\
\hline & 2 & 25.67 & $25.56(\alpha=1.100)$ & 25.60 & 25.70 \\
\hline & 3 & 25.28 & $25.15(\alpha=1.100)$ & 25.20 & 25.29 \\
\hline & 4 & 24.96 & $24.83(\alpha=1.100)$ & 24.89 & 24.96 \\
\hline \multirow{4}{*}{50} & 1 & 25.84 & $25.77(\alpha=1.075)$ & 25.79 & 25.90 \\
\hline & 2 & 25.32 & $25.21(\alpha=1.075)$ & 25.26 & 25.34 \\
\hline & 3 & 24.95 & $24.82(\alpha=1.075)$ & 24.88 & 24.95 \\
\hline & 4 & 24.66 & $24.52(\alpha=1.075)$ & 24.60 & 24.65 \\
\hline
\end{tabular}


Table 2. Fitted weights $\omega_{1}, \omega_{2}$ and $\omega_{3}$ of the parametric model (2) as functions of the noise level $\sigma$ and the amount of blur $n$ (number of convolutions with the kernel $K_{1}(3)$ ). The weights are fitted in such a way that the total sum of all group-wise MSE values inside the BM3D algorithm is minimized (5); for each group, the MSE is computed between the alpha-rooted 3-D transform coefficients of the blurry noise-free group, and the corresponding spectrum of the original group.

\begin{tabular}{|c|c|c|c|c|}
\hline$\sigma$ & $n$ (blur) & $\omega_{1}$ & $\omega_{2}$ & $\omega_{3}$ \\
\hline \multirow{4}{*}{5} & 1 & 0.0754 & 0.0060 & 0.0344 \\
\hline & 2 & 0.0918 & 0.0193 & 0.0965 \\
\hline & 3 & 0.0941 & 0.0334 & 0.1693 \\
\hline & 4 & 0.0862 & 0.0485 & 0.2244 \\
\hline \multirow{4}{*}{10} & 1 & 0.0767 & 0.0069 & 0.0379 \\
\hline & 2 & 0.0955 & 0.0209 & 0.1031 \\
\hline & 3 & 0.1061 & 0.0352 & 0.1542 \\
\hline & 4 & 0.1106 & 0.0498 & 0.1802 \\
\hline \multirow{4}{*}{15} & 1 & 0.0719 & 0.0085 & 0.0470 \\
\hline & 2 & 0.0936 & 0.0226 & 0.1022 \\
\hline & 3 & 0.1062 & 0.0375 & 0.1353 \\
\hline & 4 & 0.1083 & 0.0518 & 0.1433 \\
\hline \multirow{4}{*}{20} & 1 & 0.0673 & 0.0094 & 0.0551 \\
\hline & 2 & 0.0938 & 0.0244 & 0.0847 \\
\hline & 3 & 0.1066 & 0.0392 & 0.0955 \\
\hline & 4 & 0.1070 & 0.0535 & 0.0973 \\
\hline \multirow{4}{*}{25} & 1 & 0.0636 & 0.0111 & 0.0553 \\
\hline & 2 & 0.0878 & 0.0266 & 0.0711 \\
\hline & 3 & 0.0971 & 0.0414 & 0.0759 \\
\hline & 4 & 0.0969 & 0.0546 & 0.0777 \\
\hline \multirow{4}{*}{30} & 1 & 0.0616 & 0.0122 & 0.0469 \\
\hline & 2 & 0.0825 & 0.0280 & 0.0593 \\
\hline & 3 & 0.0809 & 0.0436 & 0.0623 \\
\hline & 4 & 0.0801 & 0.0557 & 0.0636 \\
\hline \multirow{4}{*}{35} & 1 & 0.0568 & 0.0143 & 0.0436 \\
\hline & 2 & 0.0716 & 0.0305 & 0.0497 \\
\hline & 3 & 0.0718 & 0.0446 & 0.0527 \\
\hline & 4 & 0.0675 & 0.0569 & 0.0514 \\
\hline \multirow{4}{*}{40} & 1 & 0.0519 & 0.0194 & 0.0345 \\
\hline & 2 & 0.0603 & 0.0363 & 0.0415 \\
\hline & 3 & 0.0606 & 0.0497 & 0.0453 \\
\hline & 4 & 0.0551 & 0.0615 & 0.0469 \\
\hline \multirow{4}{*}{45} & 1 & 0.0449 & 0.0153 & 0.0291 \\
\hline & 2 & 0.0511 & 0.0296 & 0.0329 \\
\hline & 3 & 0.0498 & 0.0411 & 0.0347 \\
\hline & 4 & 0.0439 & 0.0513 & 0.0359 \\
\hline \multirow{4}{*}{50} & 1 & 0.0404 & 0.0157 & 0.0260 \\
\hline & 2 & 0.0461 & 0.0296 & 0.0285 \\
\hline & 3 & 0.0446 & 0.0404 & 0.0305 \\
\hline & 4 & 0.0378 & 0.0505 & 0.0325 \\
\hline
\end{tabular}


Table 3. PSNR $(\mathrm{dB})$ of House (blurred with the PSF $K_{1} \otimes K_{1}$ ), sharpened using the model (2) and the weights in Table 2 , with seven different values for the parameter $n$.

\begin{tabular}{|c|c|c|c|c|c|c|c|c|}
\hline$\sigma$ & Spatially invariant oracle & $n=1$ & $n=2$ & $n=3$ & $n=4$ & $n=5$ & $n=8$ & $n=10$ \\
\hline 5 & $\mathbf{3 2 . 3 6}(\alpha=1.125)$ & 31.61 & 31.96 & 32.15 & 32.23 & 32.22 & 31.92 & 31.58 \\
10 & $\mathbf{3 1 . 4 9}(\alpha=1.125)$ & 30.95 & 31.24 & 31.40 & 31.47 & $\mathbf{3 1 . 4 9}$ & 31.28 & 31.02 \\
15 & $30.67(\alpha=1.125)$ & 30.31 & 30.54 & 30.67 & 30.74 & $\mathbf{3 0 . 7 7}$ & 30.70 & 30.55 \\
20 & $29.95(\alpha=1.125)$ & 29.72 & 29.92 & 30.03 & 30.09 & $\mathbf{3 0 . 1 3}$ & 30.10 & 30.01 \\
25 & $29.26(\alpha=1.100)$ & 29.14 & 29.29 & 29.38 & 29.44 & 29.47 & $\mathbf{2 9 . 4 9}$ & 29.45 \\
30 & $28.62(\alpha=1.100)$ & 28.58 & 28.71 & 28.78 & 28.82 & 28.85 & $\mathbf{2 8 . 8 9}$ & 28.88 \\
35 & $28.03(\alpha=1.075)$ & 28.05 & 28.14 & 28.20 & 28.23 & 28.26 & $\mathbf{2 8 . 3 1}$ & $\mathbf{2 8 . 3 1}$ \\
40 & $27.37(\alpha=1.050)$ & 27.45 & 27.50 & 27.53 & 27.55 & 27.56 & $\mathbf{2 7 . 5 7}$ & 27.55 \\
45 & $27.17(\alpha=1.075)$ & 27.13 & 27.20 & 27.24 & 27.27 & 27.30 & 27.36 & $\mathbf{2 7 . 3 8}$ \\
50 & $26.66(\alpha=1.075)$ & 26.67 & 26.72 & 26.75 & 26.78 & 26.80 & 26.85 & $\mathbf{2 6 . 8 7}$ \\
\hline
\end{tabular}

Table 4. PSNR (dB) of House (blurred with the PSF $K_{2} \otimes K_{2} \otimes K_{2}$ ), sharpened using the model (2) and the weights in Table 2, with seven different values for the parameter $n$.

\begin{tabular}{|c|c|c|c|c|c|c|c|c|}
\hline$\sigma$ & Spatially invariant oracle & $n=1$ & $n=2$ & $n=3$ & $n=4$ & $n=5$ & $n=8$ & $n=10$ \\
\hline 5 & $\mathbf{3 4 . 3 5}(\alpha=1.100)$ & 33.83 & 34.12 & 34.02 & 33.76 & 33.40 & 32.13 & 31.30 \\
10 & $\mathbf{3 2 . 9 6}(\alpha=1.100)$ & 32.65 & 32.88 & 32.85 & 32.72 & 32.51 & 31.64 & 31.01 \\
15 & $31.91(\alpha=1.100)$ & 31.74 & 31.92 & $\mathbf{3 1 . 9 3}$ & 31.88 & 31.78 & 31.31 & 30.93 \\
20 & $30.97(\alpha=1.100)$ & 30.90 & 31.05 & $\mathbf{3 1 . 0 8}$ & 31.06 & 31.01 & 30.72 & 30.47 \\
25 & $30.16(\alpha=1.075)$ & 30.14 & 30.27 & $\mathbf{3 0 . 3 1}$ & $\mathbf{3 0 . 3 1}$ & 30.30 & 30.15 & 30.00 \\
30 & $29.40(\alpha=1.075)$ & 29.43 & 29.52 & 29.56 & $\mathbf{2 9 . 5 8}$ & $\mathbf{2 9 . 5 8}$ & 29.51 & 29.43 \\
35 & $28.71(\alpha=1.050)$ & 28.77 & 28.85 & 28.88 & 28.90 & $\mathbf{2 8 . 9 1}$ & 28.89 & 28.85 \\
40 & $27.99(\alpha=1.025)$ & 28.07 & 28.11 & $\mathbf{2 8 . 1 2}$ & $\mathbf{2 8 . 1 2}$ & $\mathbf{2 8 . 1 2}$ & 28.08 & 28.03 \\
45 & $27.67(\alpha=1.075)$ & 27.66 & 27.73 & 27.76 & 27.78 & 27.80 & 27.84 & $\mathbf{2 7 . 8 5}$ \\
50 & $27.15(\alpha=1.050)$ & 27.17 & 27.22 & 27.25 & 27.27 & 27.28 & 27.32 & $\mathbf{2 7 . 3 3}$ \\
\hline
\end{tabular}

Table 5. PSNR (dB) of House (blurred with the PSF $K_{3} \otimes K_{3} \otimes K_{3}$ ), sharpened using the model (2) and the weights in Table 2, with seven different values for the parameter $n$.

\begin{tabular}{|c|c|c|c|c|c|c|c|c|}
\hline$\sigma$ & Spatially invariant oracle & $n=1$ & $n=2$ & $n=3$ & $n=4$ & $n=5$ & $n=8$ & $n=10$ \\
\hline 5 & $\mathbf{3 0 . 9 9}(\alpha=1.225)$ & 29.28 & 29.61 & 29.89 & 30.11 & 30.31 & 30.73 & 30.89 \\
10 & $30.10(\alpha=1.225)$ & 28.87 & 29.15 & 29.38 & 29.57 & 29.73 & 30.09 & $\mathbf{3 0 . 2 3}$ \\
15 & $29.36(\alpha=1.200)$ & 28.46 & 28.69 & 28.89 & 29.03 & 29.16 & 29.46 & $\mathbf{2 9 . 5 8}$ \\
20 & $28.66(\alpha=1.175)$ & 28.05 & 28.25 & 28.41 & 28.53 & 28.63 & 28.87 & $\mathbf{2 8 . 9 7}$ \\
25 & $27.99(\alpha=1.175)$ & 27.61 & 27.77 & 27.90 & 27.99 & 28.06 & 28.25 & $\mathbf{2 8 . 3 3}$ \\
30 & $27.41(\alpha=1.150)$ & 27.19 & 27.32 & 27.42 & 27.48 & 27.54 & 27.69 & $\mathbf{2 7 . 7 5}$ \\
35 & $26.87(\alpha=1.125)$ & 26.76 & 26.87 & 26.94 & 26.99 & 27.04 & 27.16 & $\mathbf{2 7 . 2 2}$ \\
40 & $26.35(\alpha=1.075)$ & 26.36 & 26.43 & 26.48 & 26.52 & 26.55 & 26.62 & $\mathbf{2 6 . 6 6}$ \\
45 & $26.15(\alpha=1.125)$ & 26.05 & 26.12 & 26.16 & 26.20 & 26.23 & 26.31 & $\mathbf{2 6 . 3 6}$ \\
50 & $25.72(\alpha=1.100)$ & 25.68 & 25.73 & 25.76 & 25.79 & 25.82 & 25.89 & $\mathbf{2 5 . 9 2}$ \\
\hline
\end{tabular}


Table 6. PSNR (dB) of Boat (blurred with the PSF $K_{1} \otimes K_{1} \otimes K_{1} \otimes K_{1}$ ), sharpened using the model (2) and the weights in Table 2, with seven different values for the parameter $n$.

\begin{tabular}{|c|c|c|c|c|c|c|c|c|}
\hline$\sigma$ & Spatially invariant oracle & $n=1$ & $n=2$ & $n=3$ & $n=4$ & $n=5$ & $n=8$ & $n=10$ \\
\hline 5 & $\mathbf{2 7 . 9 6}(\alpha=1.200)$ & 26.93 & 27.11 & 27.26 & 27.39 & 27.50 & 27.77 & 27.89 \\
10 & $\mathbf{2 7 . 2 7}(\alpha=1.225)$ & 26.49 & 26.64 & 26.76 & 26.86 & 26.95 & 27.17 & $\mathbf{2 7 . 2 7}$ \\
15 & $26.67(\alpha=1.225)$ & 26.09 & 26.22 & 26.32 & 26.39 & 26.46 & 26.62 & $\mathbf{2 6 . 7 1}$ \\
20 & $26.14(\alpha=1.200)$ & 25.72 & 25.83 & 25.90 & 25.96 & 26.01 & 26.14 & $\mathbf{2 6 . 2 1}$ \\
25 & $25.66(\alpha=1.175)$ & 25.38 & 25.46 & 25.52 & 25.56 & 25.60 & 25.70 & $\mathbf{2 5 . 7 6}$ \\
30 & $25.21(\alpha=1.150)$ & 25.04 & 25.11 & 25.15 & 25.19 & 25.22 & 25.29 & $\mathbf{2 5 . 3 4}$ \\
35 & $24.81(\alpha=1.125)$ & 24.72 & 24.77 & 24.81 & 24.83 & 24.86 & 24.92 & $\mathbf{2 4 . 9 5}$ \\
40 & $24.41(\alpha=1.100)$ & 24.40 & 24.43 & 24.45 & 24.47 & 24.48 & 24.52 & $\mathbf{2 4 . 5 5}$ \\
45 & $24.21(\alpha=1.125)$ & 24.13 & 24.16 & 24.18 & 24.20 & 24.21 & 24.25 & $\mathbf{2 4 . 2 8}$ \\
50 & $23.89(\alpha=1.100)$ & 23.85 & 23.88 & 23.89 & 23.90 & 23.92 & 23.95 & $\mathbf{2 3 . 9 7}$ \\
\hline
\end{tabular}

Table 7. PSNR (dB) of Boat (blurred with the PSF $\left.K_{2} \otimes K_{2} \otimes K_{2}\right)$, sharpened using the model (2) and the weights in Table 2, with seven different values for the parameter $n$.

\begin{tabular}{|c|c|c|c|c|c|c|c|c|}
\hline$\sigma$ & Spatially invariant oracle & $n=1$ & $n=2$ & $n=3$ & $n=4$ & $n=5$ & $n=8$ & $n=10$ \\
\hline 5 & $\mathbf{3 1 . 7 2}(\alpha=1.100)$ & 31.19 & 31.56 & 31.62 & 31.51 & 31.27 & 30.20 & 29.40 \\
10 & $\mathbf{3 0 . 2 4}(\alpha=1.125)$ & 29.85 & 30.11 & 30.18 & 30.17 & 30.08 & 29.55 & 29.06 \\
15 & $\mathbf{2 9 . 1 6}(\alpha=1.125)$ & 28.85 & 29.04 & 29.12 & 29.13 & 29.12 & 28.93 & 28.72 \\
20 & $28.26(\alpha=1.125)$ & 28.04 & 28.18 & 28.24 & $\mathbf{2 8 . 2 7}$ & $\mathbf{2 8 . 2 7}$ & 28.20 & 28.10 \\
25 & $27.51(\alpha=1.125)$ & 27.37 & 27.48 & 27.53 & 27.55 & $\mathbf{2 7 . 5 6}$ & 27.54 & 27.49 \\
30 & $26.87(\alpha=1.100)$ & 26.79 & 26.87 & 26.90 & 26.93 & 26.94 & $\mathbf{2 6 . 9 5}$ & 26.93 \\
35 & $26.29(\alpha=1.100)$ & 26.26 & 26.32 & 26.34 & 26.36 & 26.37 & $\mathbf{2 6 . 3 9}$ & 26.38 \\
40 & $25.67(\alpha=1.075)$ & 25.70 & 25.73 & 25.74 & 25.75 & $\mathbf{2 5 . 7 6}$ & $\mathbf{2 5 . 7 6}$ & 25.74 \\
45 & $25.41(\alpha=1.100)$ & 25.34 & 25.38 & 25.41 & 25.42 & 25.44 & 25.47 & $\mathbf{2 5 . 4 9}$ \\
50 & $25.01(\alpha=1.100)$ & 24.97 & 25.00 & 25.02 & 25.04 & 25.05 & 25.08 & $\mathbf{2 5 . 1 0}$ \\
\hline
\end{tabular}

Table 8. PSNR (dB) of Boat (blurred with the PSF $\left.K_{3} \otimes K_{3}\right)$, sharpened using the model (2) and the weights in Table 2 , with seven different values for the parameter $n$

\begin{tabular}{|c|c|c|c|c|c|c|c|c|}
\hline$\sigma$ & Spatially invariant oracle & $n=1$ & $n=2$ & $n=3$ & $n=4$ & $n=5$ & $n=8$ & $n=10$ \\
\hline 5 & $\mathbf{2 9 . 8 2}(\alpha=1.175)$ & 28.48 & 28.81 & 29.08 & 29.28 & 29.44 & 29.73 & 29.75 \\
10 & $28.62(\alpha=1.200)$ & 27.70 & 27.94 & 28.13 & 28.27 & 28.38 & 28.61 & $\mathbf{2 8 . 6 5}$ \\
15 & $27.73(\alpha=1.200)$ & 27.08 & 27.27 & 27.40 & 27.49 & 27.57 & 27.73 & $\mathbf{2 7 . 7 9}$ \\
20 & $27.01(\alpha=1.175)$ & 26.56 & 26.70 & 26.80 & 26.87 & 26.92 & 27.06 & $\mathbf{2 7 . 1 1}$ \\
25 & $26.40(\alpha=1.175)$ & 26.10 & 26.20 & 26.28 & 26.33 & 26.37 & 26.48 & $\mathbf{2 6 . 5 2}$ \\
30 & $25.86(\alpha=1.150)$ & 25.67 & 25.76 & 25.81 & 25.85 & 25.88 & 25.96 & $\mathbf{2 6 . 0 0}$ \\
35 & $25.38(\alpha=1.125)$ & 25.29 & 25.35 & 25.39 & 25.42 & 25.45 & 25.51 & $\mathbf{2 5 . 5 4}$ \\
40 & $24.90(\alpha=1.100)$ & 24.90 & 24.93 & 24.96 & 24.98 & 24.99 & 25.03 & $\mathbf{2 5 . 0 5}$ \\
45 & $24.65(\alpha=1.125)$ & 24.56 & 24.59 & 24.62 & 24.64 & 24.65 & 24.70 & $\mathbf{2 4 . 7 2}$ \\
50 & $24.30(\alpha=1.100)$ & 24.25 & 24.28 & 24.30 & 24.32 & 24.33 & 24.37 & $\mathbf{2 4 . 3 9}$ \\
\hline
\end{tabular}

